\title{
Phase Noise Effects on Wideband Mobile Radio Channel Sounding
}

\author{
Carlos Eduardo Salles Ferreira ${ }^{1}$, Gláucio Lima Siqueira ${ }^{2}$; Raimundo Sampaio Neto ${ }^{2}$. \\ ${ }^{1}$ Departamento de Engenharia de Telecomunicações, Universidade Federal Fluminense, UFF, RJ, Brazil. \\ ${ }^{2}$ Centro de Estudos em Telecomunicações, Pontifical Catholic University of Rio de Janeiro, PUC-Rio), Rio de \\ Janeiro, Brazil.
}

\begin{abstract}
In this work three wideband mobile radio channel sounding methods are simulated and compared using computational tools. The considered sounders include the traditional STDCC and two other proposed sounding methods that are based on OFDM digital transmission and detection procedures and on matched filtering. The impact of the phase noise introduced by the receiver local oscillator in the precision of the examined sounders is analyzed by comparing the estimated channel results with the simulated reference channel. Other impairments factors like thermal noise or nonlinear distortion affecting the system are not considered.
\end{abstract}

Index Terms - Matched filter, OFDM, Phase noise, STDCC, wideband mobile radio channel sounding.

\section{INTRODUCTION}

The choice of modulation scheme and the analytical evaluation of performance of communication systems rely strongly on the satisfactory characterization of the transmission channels. Therefore, it is important to characterize the time varying random channels typical of radio systems. In communications where radio links are employed, the channels, especially mobile radio channels, though linear, show a behavior that varies with time. In these scenarios, because the transmitter or receiver or both dislocate, the channels are highly variable, mainly in urban environments where the scatters cause multipath and Doppler Effects. To make communication viable, countermeasures are inserted, such as the use of diversity and equalizers [1], as well as the choice of adequate modulation methods and transmission rates. The characterization of mobile radio channels can be performed either in narrowband or wideband channels with a sounder, a set of equipment made up of a transmitter and receiver and their respective aerial systems (transmission lines, antennas, etc.), which is capable of estimating mobile radio channel parameters.

This work focuses on the effects of phase noise on wideband sounders. The considered sounders include the STDCC (swept time-delay cross-correlation) traditional sounding method [10] and two other methods proposed and used here as alternative sounding techniques. One is based on OFDM (Orthogonal Frequency-Division Multiplexing) digital transmission and detection procedures and the other relies on matched filtering processing, where the filter is matched to the transmitted test sequence. Aiming at increasing the precision of the sounding results, the proposed methods collect, 
and adequately process, more than one sample per received test sequence symbol [2]. Matlab® and Simulink ${ }^{\circledR}$ computational tools were used. Section II presents an introduction to phase noise models and characteristics. The traditional model of the STDCC [3] and the simulation of the proposed Matched Filter and OFDM based sounders are described in Section III. Theoretical aspects supporting the last two methods are presented in the Appendix. The raw data produced at the sounders output by the Simulink ${ }^{\circledR}$ simulations were processed and the results are presented in Section IV. Section V discusses the values of the RMS error and standard deviation derived from the results shown in section IV. Finally, section VI presents the conclusion.

\section{PHASE NOISE MODEL}

Probably the most known model to represent the phase noise was proposed by Leeson [4] where the output of a stable oscillator is represented by

$$
v(t)=A \cos \left[\omega_{0} t+\varphi(t)\right]
$$

where $\varphi(t)$ is a zero-mean stationary random process representing phase shift from its ideal value. Based on this model the well-known Leeson's equation was developed.

Despite the fact that Leeson had classified his model as "a heuristic derivation presented without formal proof", Sauvage [5] mentions that Leeson's model was the result of a logical physical reasoning, and developed a mathematical demonstration for the model. An important aspect to be mentioned is the good match between practical results and the predicted values obtained by Leeson's model.

The Leeson's model is based on the following premises considering the set amplifier and feedback filter as the oscillator core.

- The positive feedback circuit is an RLC with load factor equal to $Q_{l}$;

- The oscillator uses a linear amplifier;

- Within the $3 \mathrm{~dB}$ bandwidth, the phase error produced by the thermal noise results in a frequency error defined by phase-frequency relation;

- Outside the $3 \mathrm{~dB}$ feedback filter bandwidth; there is no more feedback effect, but only internal noise contributions.

Considering these premises Sauvage [5] proposed that the phase noise power spectral density, $\mathcal{L}_{\varphi}(\omega)$, existing in the oscillator output is represented by:

$$
\mathcal{L}_{\varphi}(\omega)=L_{i}(\omega)|H(j \omega)|^{2}
$$

where $L_{i}(\omega)$ is the phase noise power spectral density produced by internal circuit imperfections and $|H(j \omega)|^{2}$ is the RLC resonator transfer function. $L_{i}(\omega)$ is assumed to follow a $\omega^{-1}$ law below the flicker frequency $\omega_{c}$. Beyond $\omega_{c}, L_{i}(\omega)$ presents a behavior arising from internal white noise 
following $\omega^{0}$ law. When the oscillator has a high quality factor, $Q$, it is possible to consider $\omega_{0} / 2 Q<\omega_{c}$. In this case, Fig. 1a represents $L_{i}(\omega)$, Fig. 1b represents $|H(j \omega)|^{2}$ and Fig. 1c represents the results of mathematical operation specified in (2).
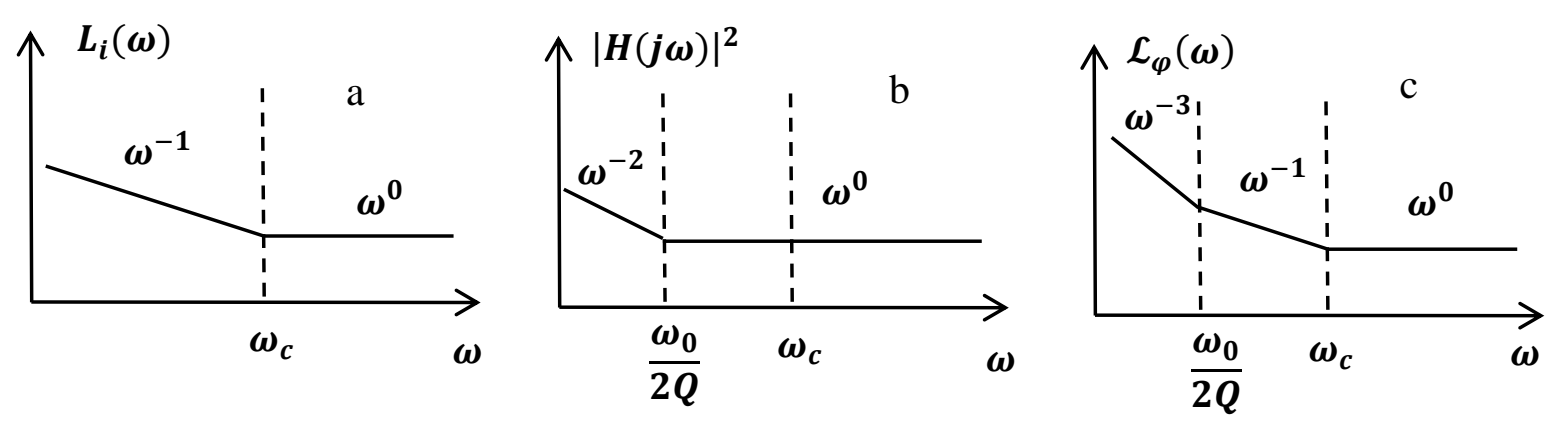

Fig. 1. Leeson's model for high Q oscillator. Logarithmic scales

Leeson's equation for only one feedback circuit is expressed by:

$$
\mathcal{L}\left(\omega_{m}\right)=10 \log \left[\frac{1}{2}\left(\left(\frac{\omega_{0}}{2 Q_{l} \omega_{m}}\right)^{2}+1\right)\left(\frac{\omega_{c}}{\omega_{m}}+1\right)\left(\frac{F k T}{P_{s}}\right)\right]
$$

where:

$\mathcal{L}\left(\omega_{m}\right)$ : Single-sideband phase noise density $(\mathrm{dBc} / \mathrm{Hz})$

$F$ : Noise factor of the amplifier;

$k$ : Boltzmann's constant;

T: Absolute temperature (K);

$P_{S}$ : Oscillator output power $(\mathrm{W})$;

$Q_{l}$ : Loaded $Q$ (dimensionless);

$\omega_{0}$ : Oscillator output angular frequency $(\mathrm{rad} / \mathrm{s})$;

$\omega_{c}$ : Corner angular frequency of flicker noise $(\mathrm{rad} / \mathrm{s})$;

$\omega_{m}$ : Angular frequency off-set from the carrier $(\mathrm{rad} / \mathrm{s})$.

The region of $\mathcal{L}_{\varphi}(\omega)$ characterized by the decrease rate of $\omega^{-1}$ is used at Matlab® to simulate the phase noise to be introduced in systems using the model proposed by Kasdin [7].

For OFDM systems, Armada [8] mentions that a value of phase noise variance equal to $1.33 \mathrm{rad}^{2}$ introduces an equivalent degradation between $10 \mathrm{~dB}$ and $25 \mathrm{~dB}$ in $E b / N_{0}$ ratio in real systems. Therefore, such variance is not recommended to be used in OFDM systems. From phase noise versus off-set frequency curves presented by Armada [8], this variance value is equivalent to $-40 \mathrm{dBc}$ at 100 $\mathrm{Hz}$ off-set frequency. The maximum phase noise value used in the simulations for all compared methods was $-35 \mathrm{dBc}$, which is $5 \mathrm{~dB}$ worse than $-40 \mathrm{dBc}$.

Thus, an off-set frequency equal to $100 \mathrm{~Hz}$ was chosen and applied using the following phase noise levels for all compared methods: $-35 \mathrm{dBc},-40 \mathrm{dBc},-45 \mathrm{dBc},-50 \mathrm{dBc},-60 \mathrm{dBc},-70 \mathrm{dBc},-80 \mathrm{dBc},-90$ $\mathrm{dBc},-120 \mathrm{dBc}$. This phase noise range includes best, worse and typical values usually found in equipment. 


\section{OFDM, MATCHED FILTERING AND STDCC BASED SOUNDING METHODS}

\section{A. OFDM Sounder}

Fig. 2 presents a baseband complex equivalent block diagram of the proposed OFDM sounder. For transmission, a pseudo-noise (PN) sequence of length $M$ with an added zero is initially generated. Assuming BPSK (Binary Phase Shift Keying) modulation, the test sequence, with an equal number of zeroes and ones, is mapped into the test sequence with values of \pm 1 . This sequence is transformed through an IDFT (Inverse Discrete Fourier Transform) using an IFFT (Inverse Fast Fourier Transform) algorithm of length $M$. Then, a square-root-raised-cosine transmission shaping filter is introduced, generating the transmitted analog signal. In the implementation employing Simulink ${ }^{\circledR}$, the transmission shaping filter has an oversampling factor $S$, and from that, a signal sampled in discrete time is processed.

In the actual implementation of the receiver, the band pass analog signal is received, converted to baseband, filtered by a square-root-raised-cosine reception filter and sampled at twice the symbol rate, resulting in two samples per received symbol. This operation is performed in Simulink ${ }^{\circledR}$ by using a downsampling factor of $S / 2$ in the square-root-raised-cosine reception filter.

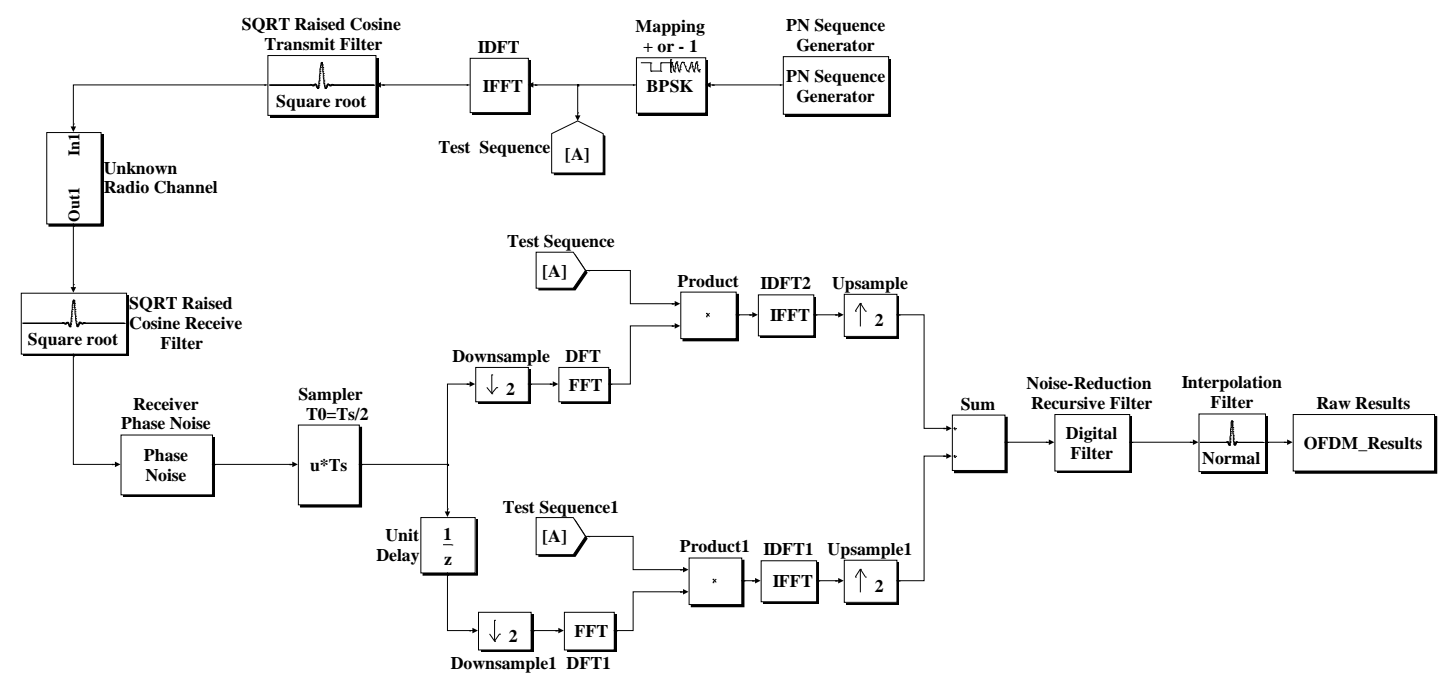

Fig. 2. Baseband complex equivalent block diagram of the OFDM sounder

From there, the signal is split into two branches. In one branch, a symbol delay is introduced and the sequence of samples is downsampled by a factor of two. In the other branch, no delay is introduced, and the signal is subsampled by the same factor. Consequently, the first branch contains the even numbered samples of the signal, while the second contains the odd numbered samples. Next, a normalized DFT (Discrete Fourier Transform) is applied to the signals in each branch using a size $M$ FFT (Fast Fourier Transform) algorithm.

To estimate the response of the sounded channel in the frequency domain, the size $M$ output of the FFT block (i.e., the received signal in the time domain with all the distortions introduced by the radio 
channel with their multipath) is divided, element by element, by the \pm 1 components of the test sequence. Two frequency domain channel representations are thus obtained - one for the odd numbered samples, and other for the even numbered samples.

To obtain the channel impulse response estimate, the IDFT is applied once more to the odd and even sample results. After oversampled by a factor of two, the sequences are then added, with one shifted one position in time, to finally obtain an estimated channel impulse response with twice the number of samples. Fig. 3 shows the signals obtained in the time domain in the upper and lower branches, respectively and the final result for a simulated channel with three paths, wherein the delay of the second path is an integer multiple of the test symbol duration, $T s$, and the delay of the third path is a non-integer multiple of this duration.
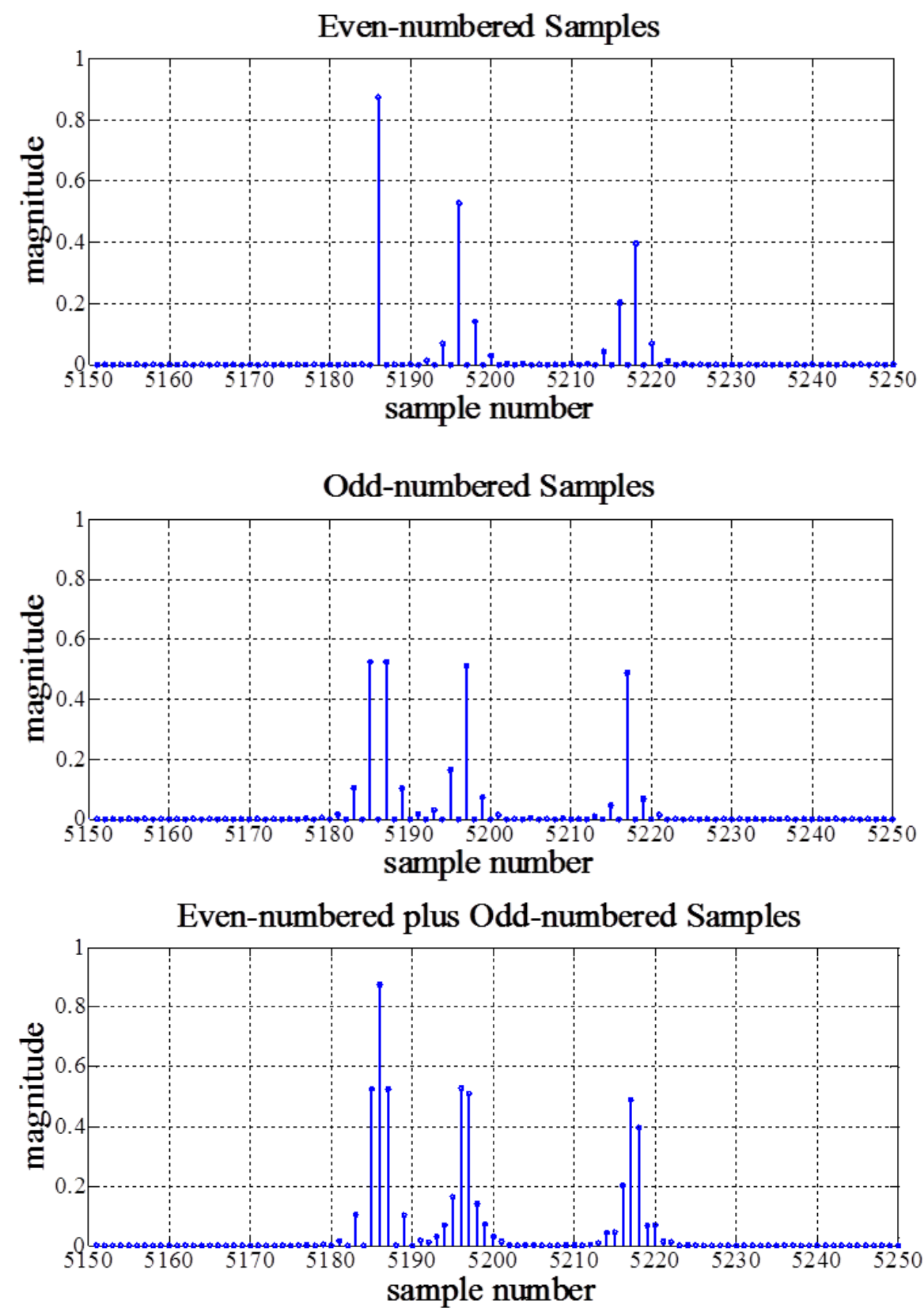

Fig. 3. Even-numbered samples (top), odd-numbered samples (middle) and resulting channel magnitude from the sum of even and odd-numbered samples (bottom) 
As the last processing stage to obtain raw data, a recursive low-pass filter can be inserted, with the objective of reducing the effects of the additive receiver noise (not considered here).

Finally, intermediate values are interpolated from those obtained by inserting a raised cosine lowpass filter, similar to the ones used in pulse shaping, with an oversampling factor $S$.

Fig. 3 (bottom) and 4 present the images of signals obtained through Simulink® before and after interpolation.

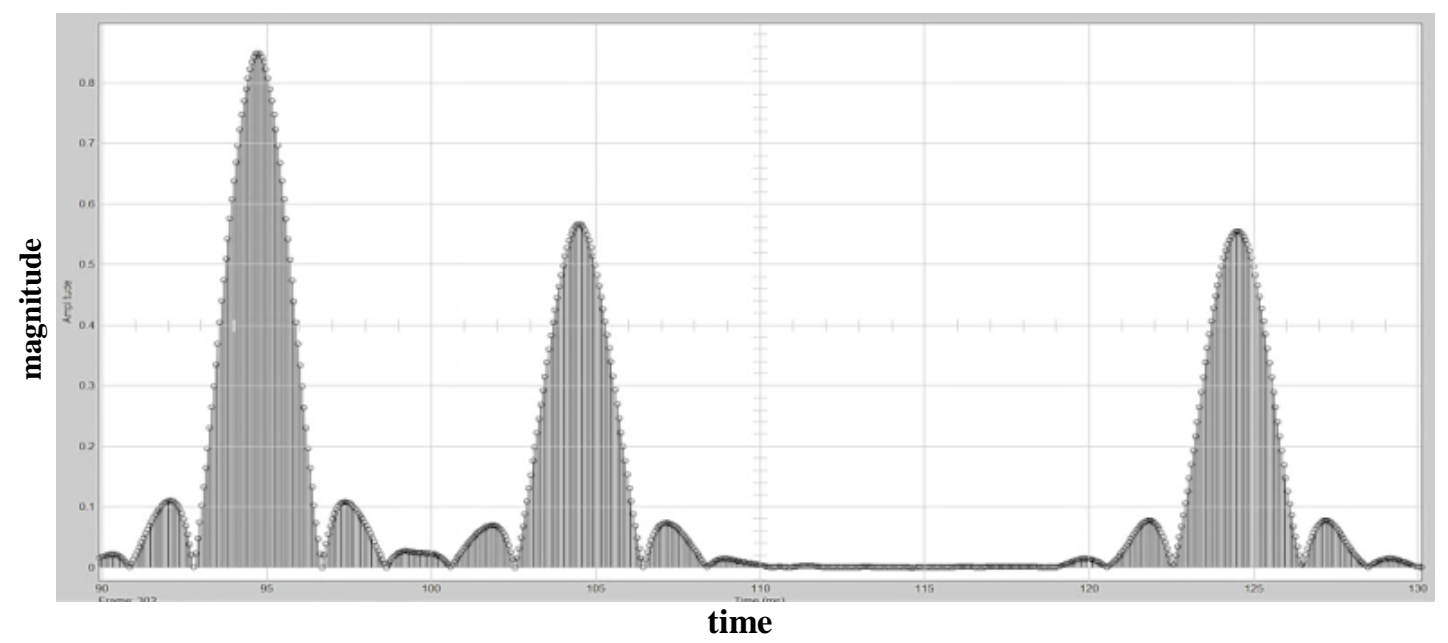

Fig. 4. Interpolator filter effect: OFDM generated estimates - magnitude

\section{B. $\quad$ Matched Filter Sounder}

Fig. 5 presents a baseband complex equivalent block diagram of the proposed Matched Filter sounder. In the Simulink® implementation, as in the OFDM section, the signal passes through the square-root-raised-cosine pulse shape reception filter and has two samples collected from each received symbol.

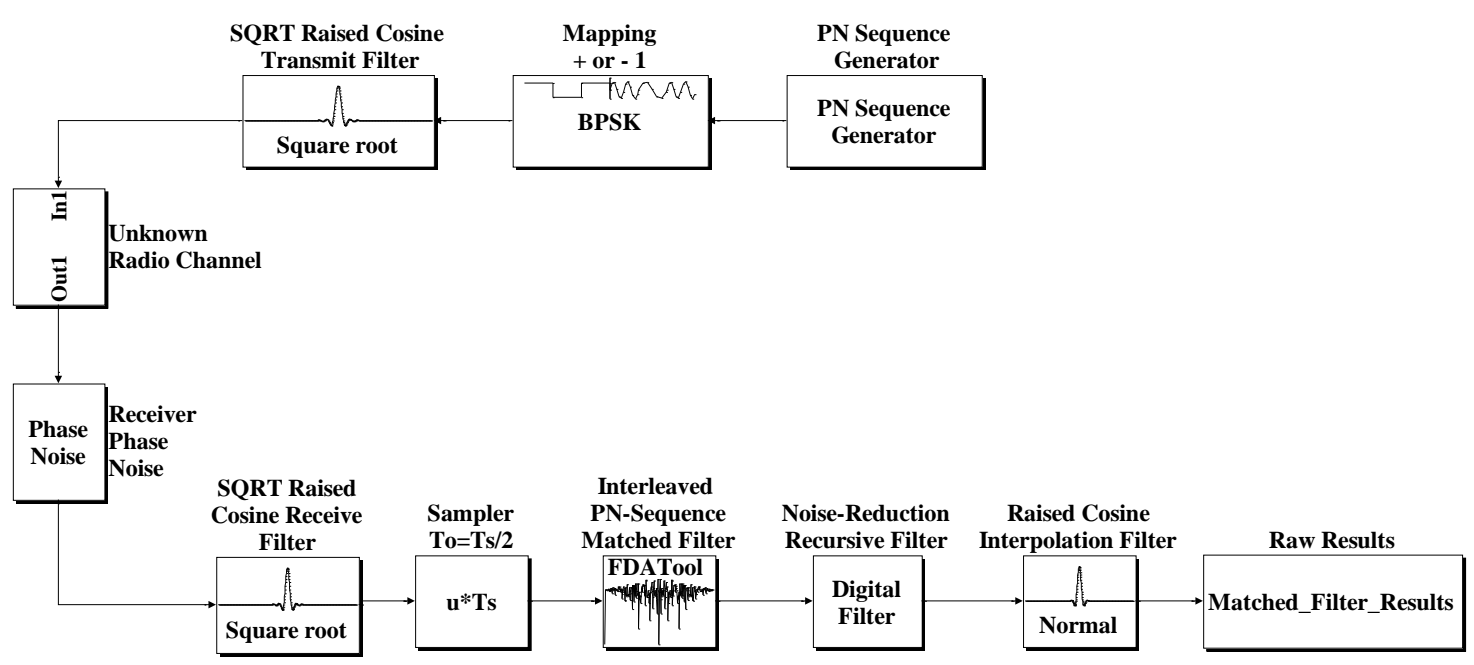

Fig. 5. Baseband complex equivalent block diagram of the Matched Filter sounder

In the matched filter implementation the coefficients of the filter matched to the test sequence are interleaved with zeroes, thus resulting in a FIR (Finite Impulse Response) filter of length $2 \times N$, 
referred to as an interleaved matched filter. Fig. 6 represents the structure of this filter.

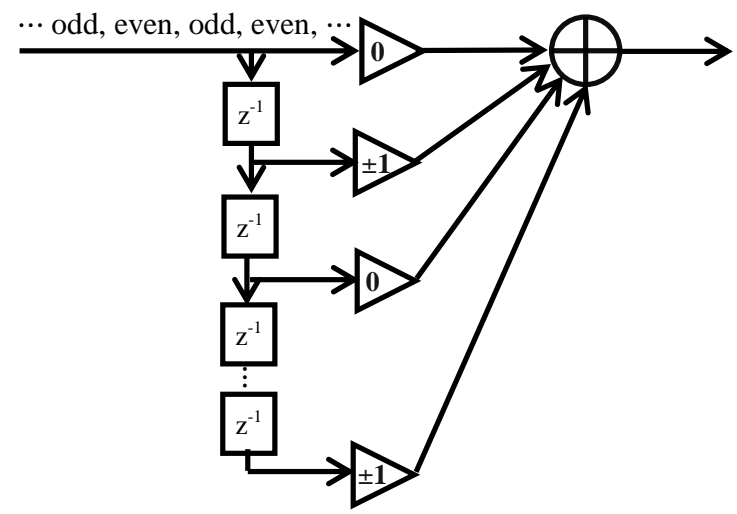

Fig. 6. FIR interleaved matched filter

Therefore, for every pulse of the clock, there is a shift in the sequence of samples of the received signal inside the interleaved matched filter, so that the samples are filtered, in turns, by the filter matched to the original test sequence; first the even numbered samples, and then the odd numbered ones. Thus here, as in the proposed OFDM sounder, the number of samples in the channel estimate is also doubled. It is important to emphasize that this zero interleaving procedure can be used for higher number of samples per symbol.

The processing of the signal at the output of the interleaved matched filter is identical to the one for the OFDM sounder. After the interpolation procedure, a file with the results is generated for future processing. Figures 3 (bottom) and 4 used in the description of the OFDM sounder are valid to represent the Matched Filter signal before and after interpolation. In Fig. 7, a single path channel estimate generated by the Matched Filter sounder is shown, with and without the use of the interleaved filter (one sample and two samples per symbol respectively), where the result obtained with the interleaved filter appears on the right side of the image.

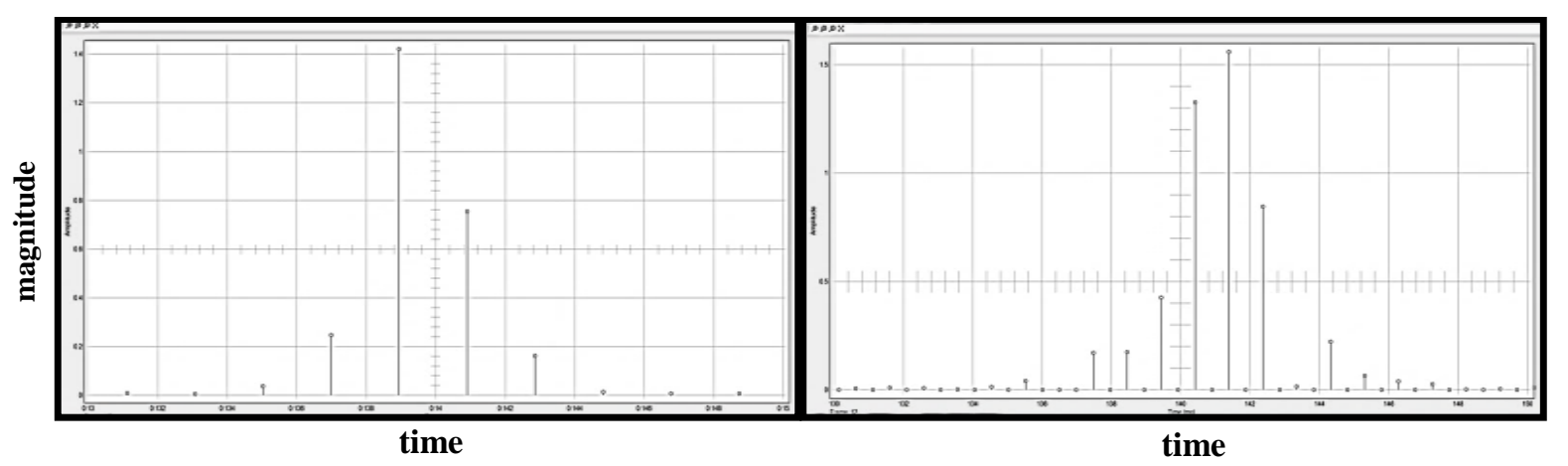

Fig. 7. Estimated channel magnitude - Results obtained with one sample per symbol (left) and two samples per symbol (right)

Fig. 8 displays an image obtained with results generated by the Matched Filter sounder, showing 
the estimated channel modulus in one simulation. In the rising part of the envelope, the recursive filter convergence is noted. After convergence the sounder tracks the time variations of the channel magnitude. A new channel estimate is obtained for each test sequence received. The lower part of Fig. 8 shows a closer look at the channel estimates generated during and after convergence of the noise reduction IIR filter. An image similar to Fig. 8 was obtained with the OFDM method.
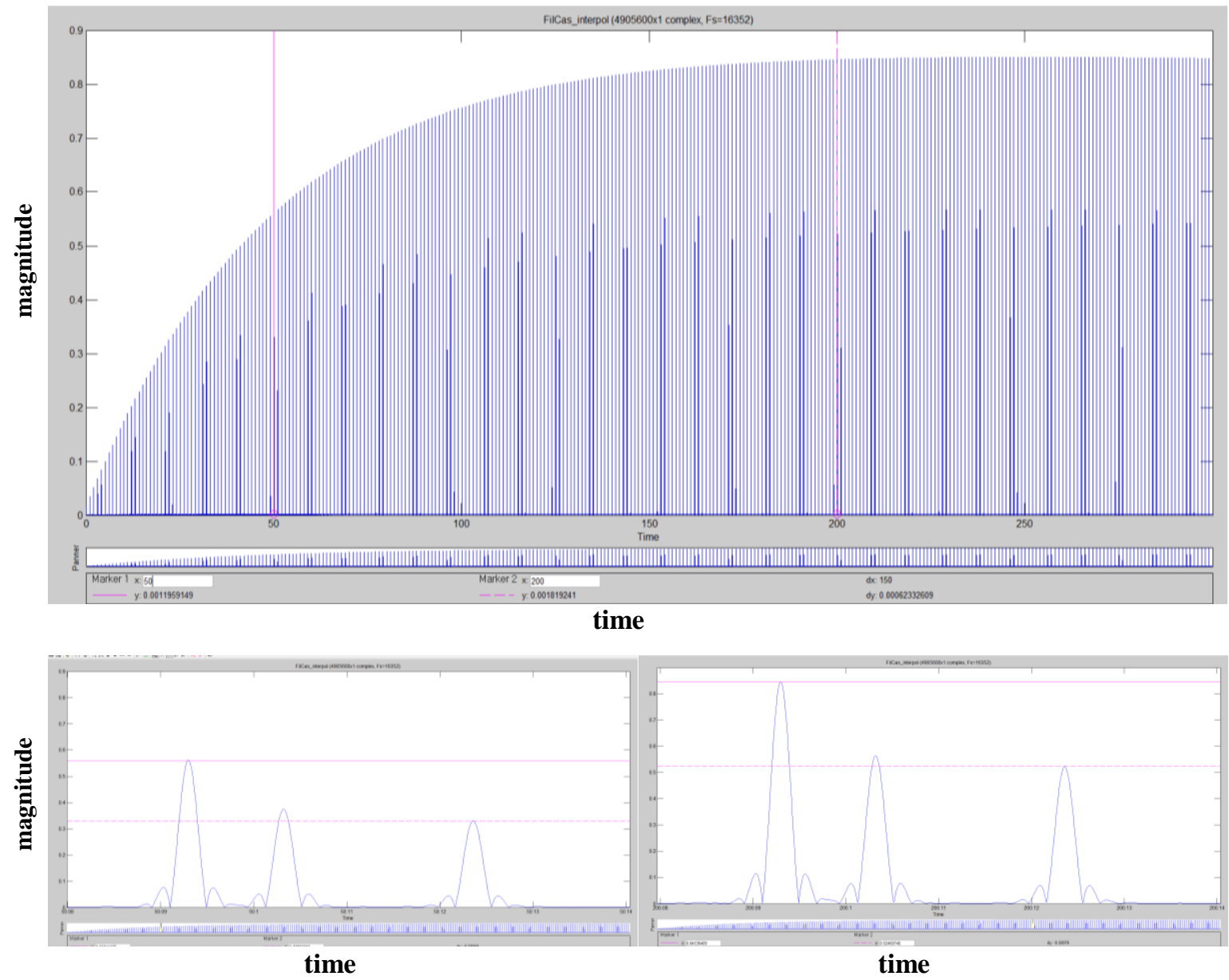

Fig. 8. Channel estimates generated by the Matched Filter sounder- Details during and after convergence of the IIR filter.

\section{STDCC Sounder}

Fig. 9 shows the equivalent baseband block diagram of the implemented wideband channel sounder using spectrum scattering, known as STDCC (swept time-delay cross-correlator). It should be noted that the STDCC is an analog traditional method used for radio channels estimations [3] [10].

In the STDCC method, the carrier is spread over a frequency band by a pseudo-noise (PN) test sequence signal of length $\mathrm{M}$. This sequence has a bit period equal to $T_{c}$ and a bit rate $R_{c}$ equal to $1 / T_{c}$. 


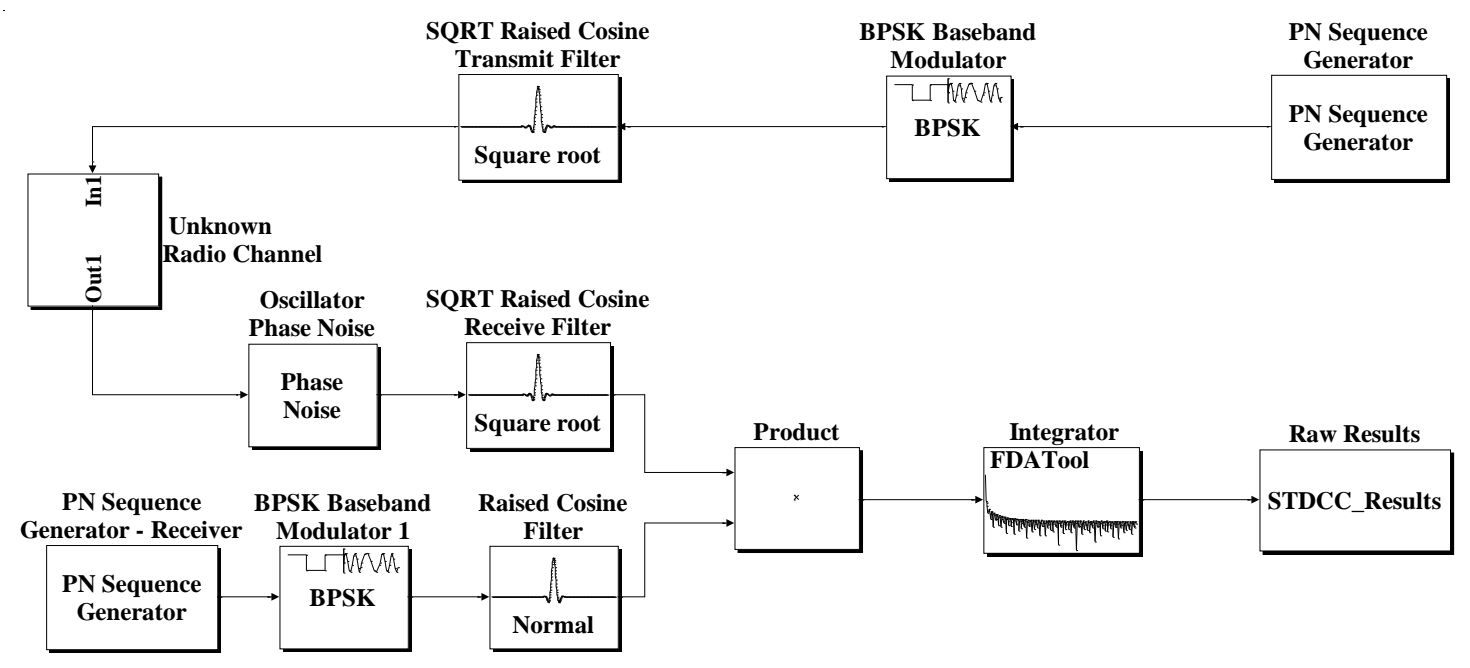

Fig. 9. Baseband complex equivalent block diagram of the STDCC sounder

In this method, the received scattered signal is correlated with an identical PN sequence signal generated in the receiver. Although the two sequences have identical content, the clock that determines the bit rate of the transmitter has a slightly higher frequency than the receiver. A sliding correlator is implemented mixing and filtering the two sequences. When the higher rate transmitted PN sequence aligns with the lower rate receiver generated sequence, a correlation peak occurs.

The STDCC method has an equivalent time measurement, which is updated every time the two sequences are correlated to the fullest. The elapsed time between two adjacent maximum correlations $\Delta T$ is calculated by:

$$
\Delta T=T_{c} \gamma M=\gamma M / R_{c}
$$

where:

$T_{c}$ : bit period (s);

$R_{c}$ : bit clock frequency $(\mathrm{Hz})$;

$\gamma$ : slide factor (dimensionless);

$M$ : PN sequence length (bit)

The slide factor $\gamma$ is defined as the relationship between the rate of transmission bit clock and the difference between clock rates of transmission and reception. Mathematically is expressed as:

$$
\gamma=\alpha /(\alpha-\beta)
$$

where,

$\alpha$ : transmitter bit clock rate $(\mathrm{Hz})$;

$\beta$ : receiver bit clock rate $(\mathrm{Hz})$.

It should be noticed that in the time period required by the STDCC method to generate one single channel estimate result, the other two methods produces $\gamma$ channel estimates. 


\section{PRIMARY CALCULATED PARAMETERS}

The simulation was conducted for an ensemble of ten sample functions of phase noise for each of nine different values of the ratio carrier power to the power spectral density of phase noise at a given off-set frequency. Final results are an average over the considered ensemble.

The simulated channel used as a reference is selective both in time and in frequency. The selectivity in time is associated with the Doppler spread while the selectivity in frequency is associated with time scattering arising from resolved multipath. The simulation considered an $\mathrm{N}$-path channel. The amplitudes of the paths were modeled as independent complex wide-sense stationary, Gaussian processes with power spectral densities given by the Jakes model [11]. This model has the Doppler frequency $f_{0}$ as a parameter and is frequently used in mobile communication scenarios. The description of the simulated channel model used by Matlab® is presented in [9].

After obtaining the channel estimation results, the three methods were compared computing the ratio between the estimated channel ratio and the reference channel ratio according to.

$$
\begin{aligned}
& R(n, k)=\frac{\frac{\text { Complex Amplitude of Estimated Path }(n)}{\text { Complex Amplitude of Estimated Path }(k)}}{\frac{\text { Complex Amplitude of Reference Path }(n)}{\text { Complex Amplitude of Reference Path }(k)}}= \\
& =|R(n, k)| e^{-\mathrm{j} \Theta(n, k)}
\end{aligned}
$$

where $2 \leq n \leq N, \quad$ and $n>k$.

In (6), $N$ denotes the total number of existing paths in the search environment, and $n$ and $k$ represent the arriving order of the path at the sounder mobile receiver. Since the coefficients of the estimated channel and the reference channel, are, in general, complex the ratio $R(n, k)$ defined in (6) has modulus, $|R(n, k)|$, and phase $\Theta(n, k)$. An ideal estimation would result in $|R(n, k)|=1$ and $\Theta(n, k)=0$.

For the simulated radio channel to be considered approximately time invariant the value of product $f_{D} \times T$ was assumed equal to $10^{-4}$, where $f_{D}$ represents the maximum Doppler shift and $T$ is the test sequence period.

The reference mobile channel has $N=3$ paths allowing the computation of three ratios: path2/path1, $R(2,1)$, path3/path1, $R(3,1)$ and path3/path2, $R(3,2)$. This last ratio, in fact, presents an information redundancy since it can be obtained from the other two ratios. The adopted delay between path 1 and path 2 is an integer multiple of the symbol time interval while the adopted delay between path 1 and path 3 is a non-integer multiple of the symbol time interval. 


\section{NUMERICAL RESULTS}

Fig. 10 presents modulus and phase results obtained from (6) for each simulated phase noise value.
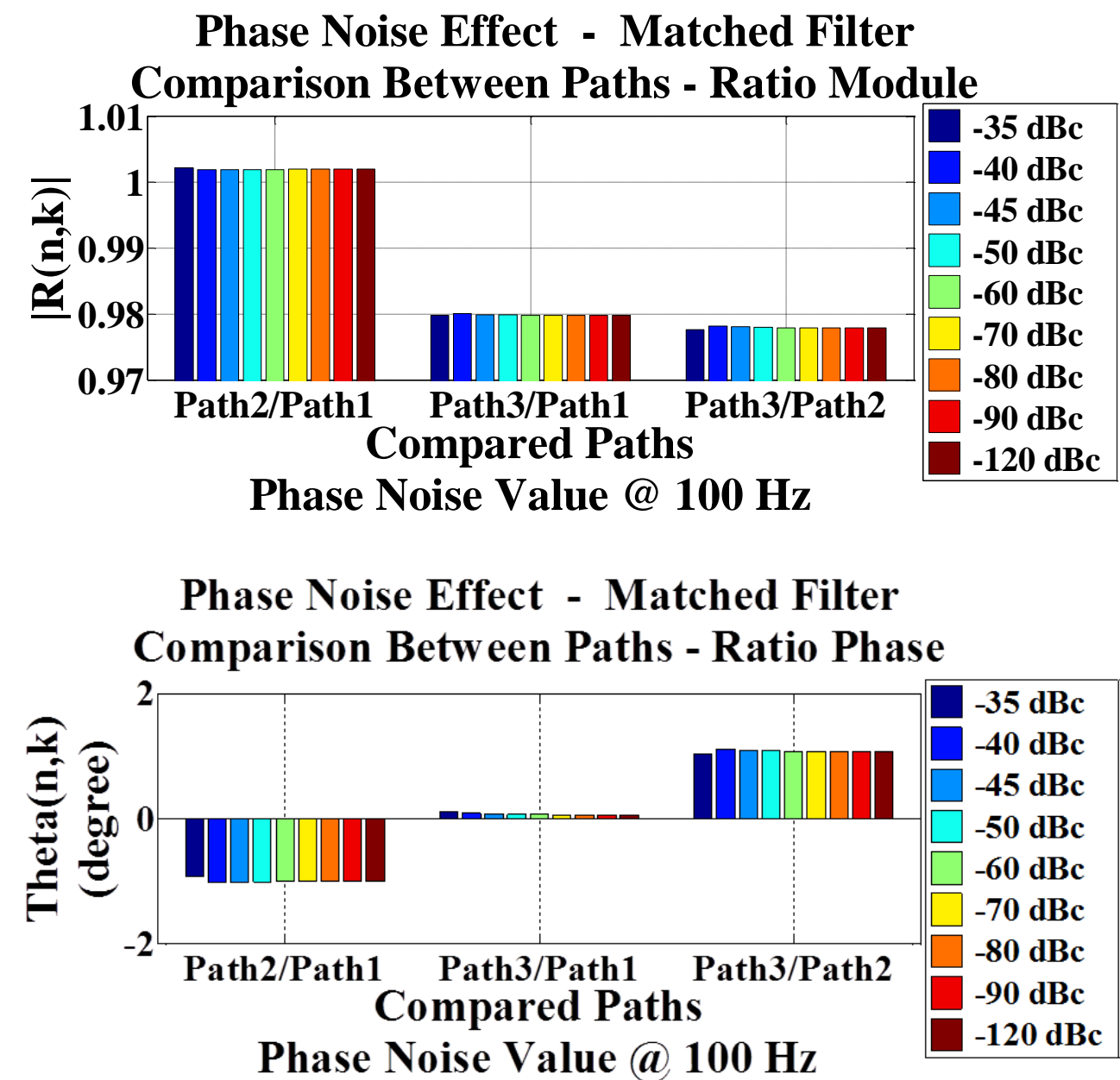

a) Matched Filter

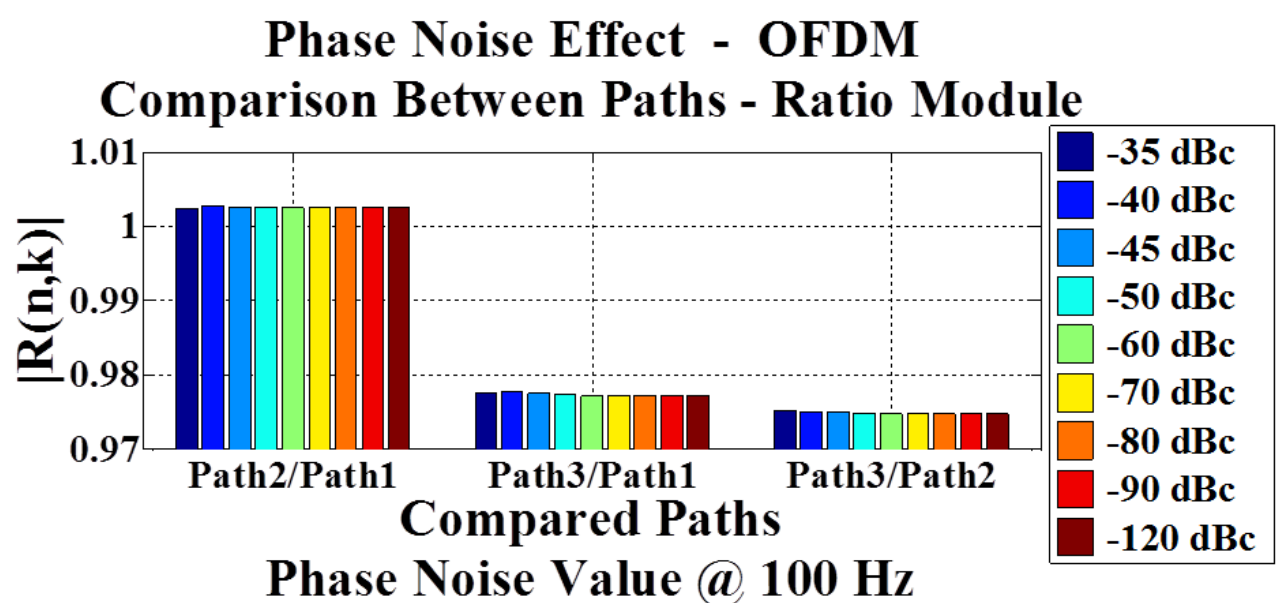




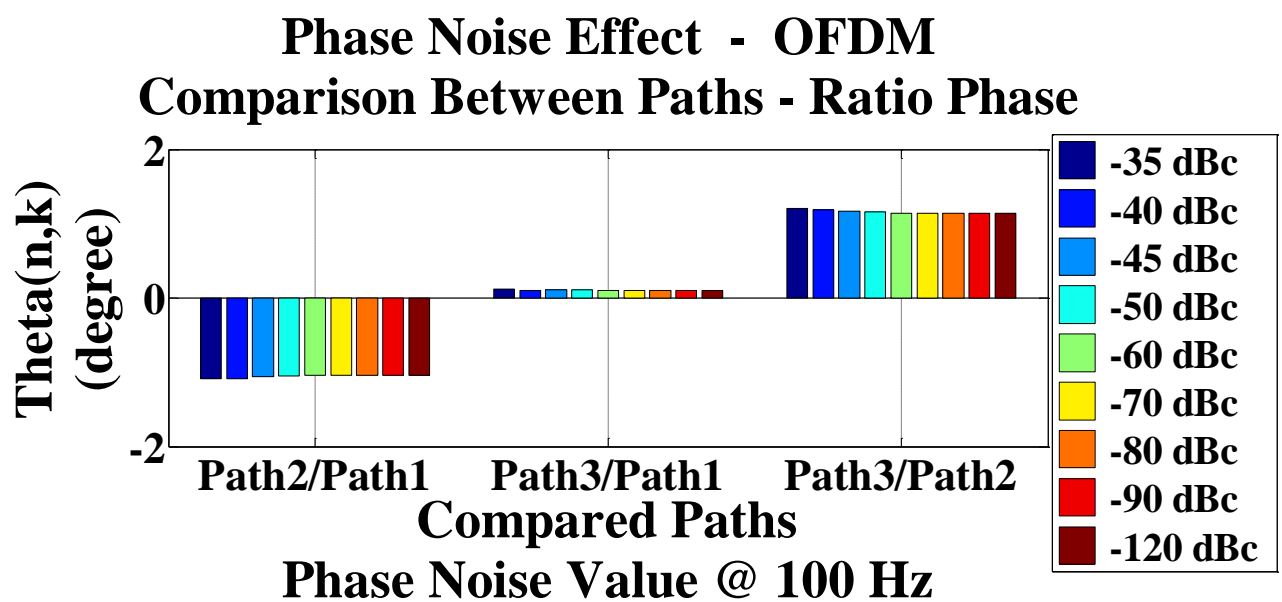

b) OFDM

Phase Noise Effect - STDCC

Comparison Between Paths - Ratio Module

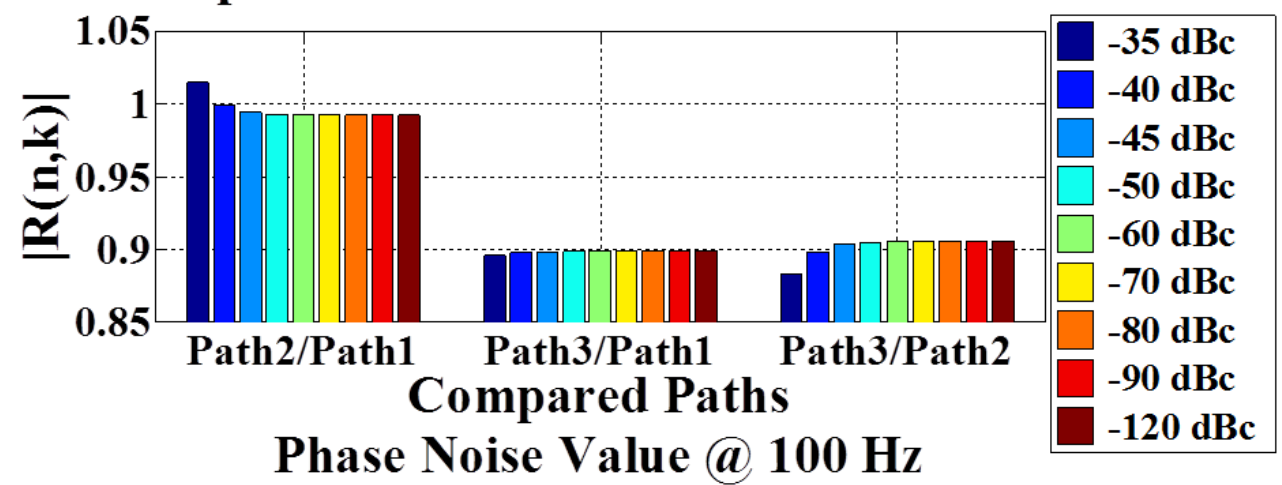

Phase Noise Effect - STDCC

Comparison Between Paths - Ratio Phase

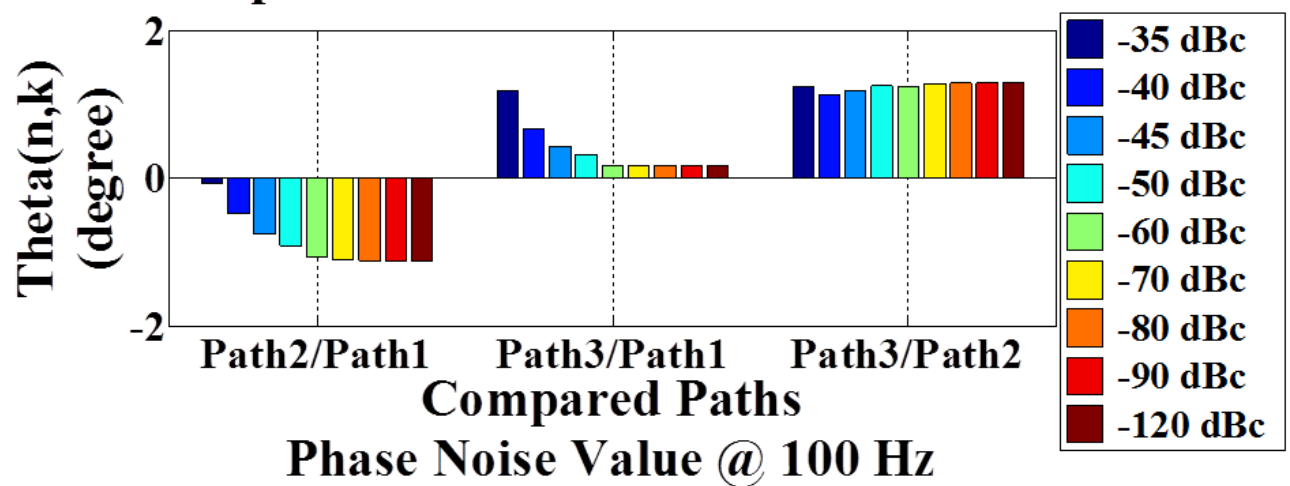

(c) STDCC

Fig. 10. Results after use of (6) for the three methods

From the results shown in Fig. 10 both the precision and the dispersion were analyzed for each method according to the calculated parameters. 
- RMS value of the ratio error, $|1-R(n, k)|$;

- Standard deviation of the ratio modulus, $|R(n, k)|$;

- $\quad$ RMS value of the complex ratio phase, $\Theta(n, k)$;

- Standard deviation of the ratio, phase, $\Theta(n, k)$.

The RMS error and standard deviation results were computed over nine different simulated values of phase noise.

Fig. 11 shows the ratio RMS error. Notice a similar performance between Matched Filter and OFDM methods. Both have a very precise result considering the path2/path1 ratio and less than 2.5\% error when path3 is computed. Also the STDCC method presents a better performance when estimating the path2/path1 ratio, however four times worse than Matched Filter method (0.008/0.002). Again the STDCC method is worse than the others when path 3 is considered, showing an estimated RMS error of about $10 \%$.

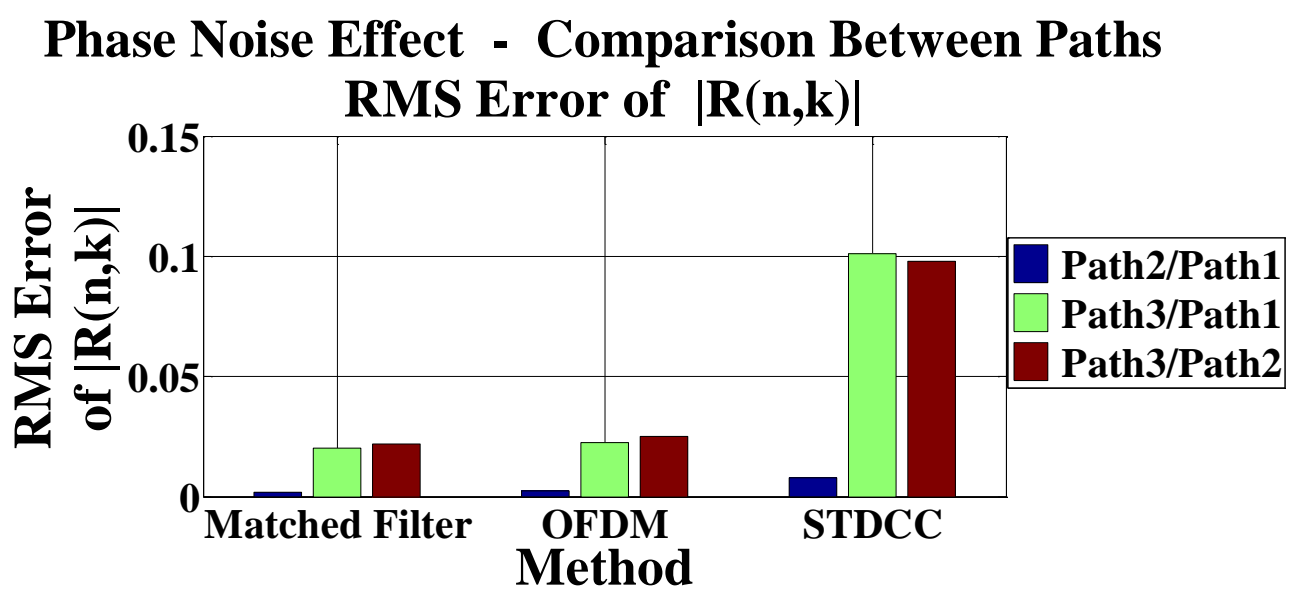

Fig. 11. RMS error of $|\boldsymbol{R}(\boldsymbol{n}, \boldsymbol{k})|$

Fig. 12 shows the Matched Filter as the method with lower sensibility to variations in the phase noise levels closely followed by the OFDM method. But, interestingly, in STDCC method the path2/path1 ratio is more susceptible to the presence of phase noise. Fig. 10 (c) shows that between $35 \mathrm{dBc}$ and $-60 \mathrm{dBc}$ the phase noise changes the STDCC results considerably but only a small effect is observed for lower $\mathrm{dBc}$ values. 


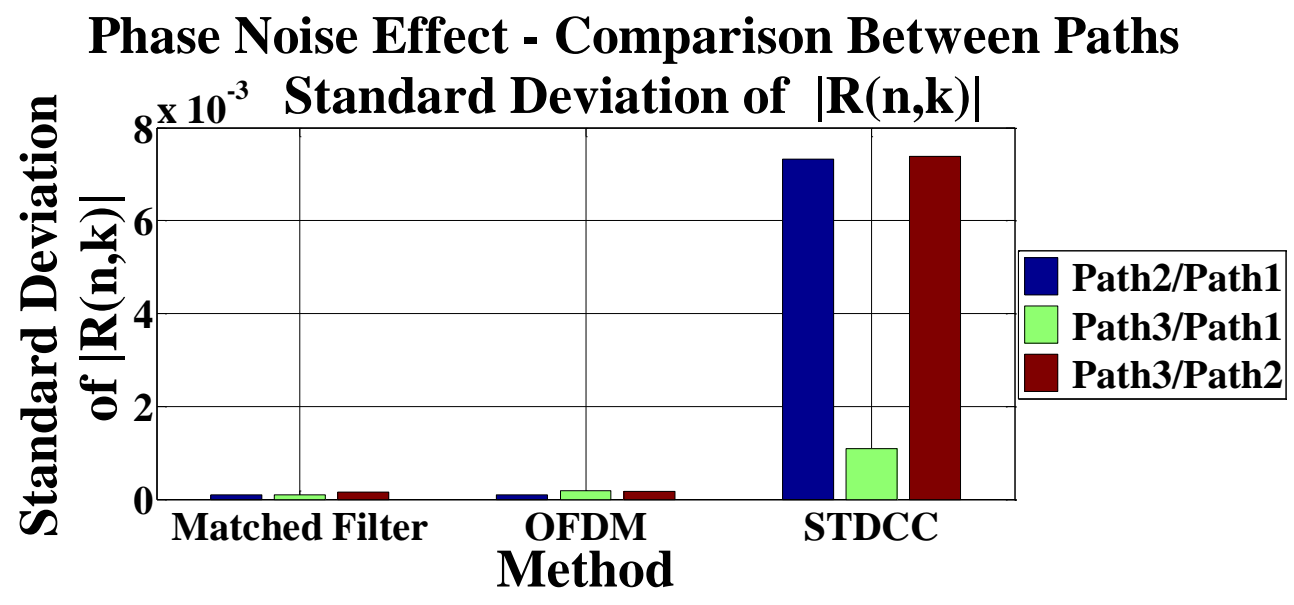

Fig. 12. Standard deviation of $|\boldsymbol{R}(\boldsymbol{n}, \boldsymbol{k})|$

The RMS values of $\Theta(n, k)$ presented in Fig. 13 indicate that variations in the phase noise levels have small impact on precision of the methods. The maximum detected value is approximately $1.3^{\circ}$.

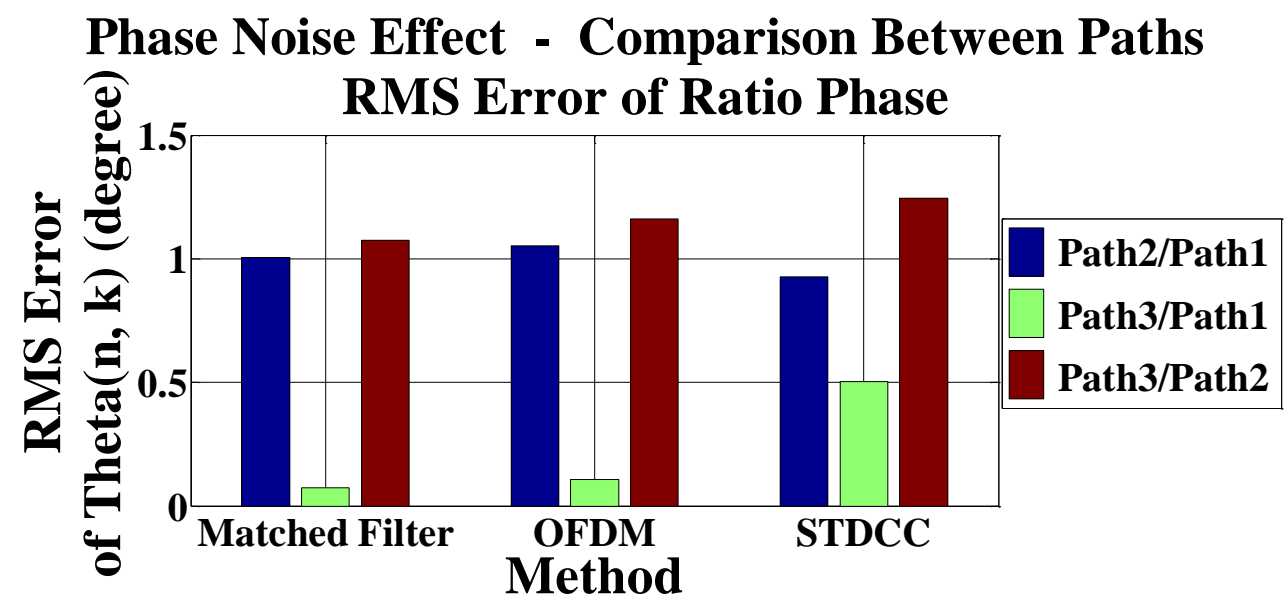

Fig. 13. RMS error of $\Theta(\mathbf{n}, \mathbf{k})$

Still considering the ratio $R(n, k)$ phase error, $\Theta(n, k)$, from the point of view of sensitivity to phase noise level variation indicated by the $\Theta(n, k)$ standard deviation values of Fig. 14, the STDCC method is the worse, when compared with the other two methods. However, the standard deviation values are all very small with estimated value of about $0.4^{\circ}$. 


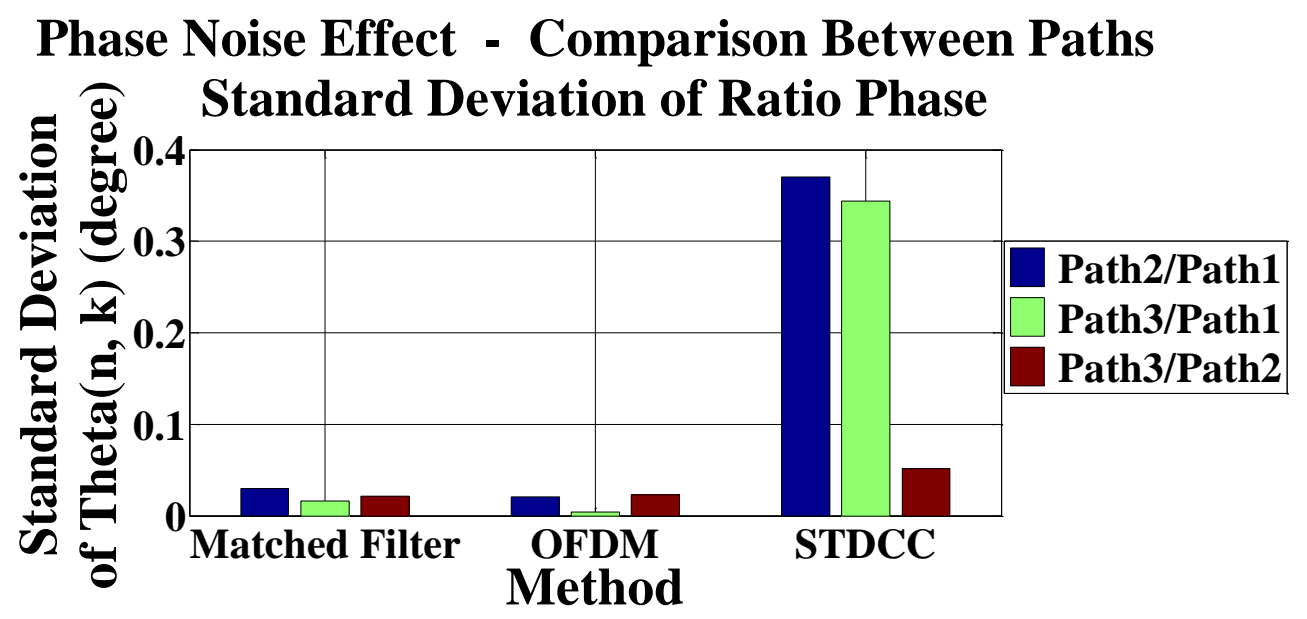

Fig. 14. Standard deviation of $\boldsymbol{\Theta}(\boldsymbol{n} . \boldsymbol{k})$

\section{CONCLUSION}

In this work three wideband mobile radio channel sounding methods were simulated using computational tools. The implemented sounders included the traditional STDCC and two other proposed sounding methods, based on OFDM digital transmission and detection procedures and on matched filtering. The impact of the phase noise introduced by the local oscillator in the precision of the examined sounders was analyzed by comparing the simulated reference channel with the estimated channel results for several different phase noise levels. Other impairments factors like thermal noise or nonlinear distortion affecting the system were not considered. The OFDM and Matched Filter methods collect and process more samples per received symbol aiming at increasing precision of the estimation method before interpolation

Considering the ratio $R(n, k)$ error, $|1-R(n, k)|$, the Matched Filter and OFDM methods presented RMS values smaller than 2.5\%, while the STDCC results reached $10 \%$ for the path3/path1 ratio. The results for the standard deviation of $|R(n, k)|$ indicated the STDCC method as the most sensitive to phase noise levels variations, even though the higher estimated value, $0.8 \%$, is not relevant in field measurements. Concerning the values of the ratio $R(n, k)$ phase, $\Theta(n, k)$, the STDCC method was also the less reliable, although the RMS error and standard deviation results presented negligible values for all compared methods.

An analysis of the results shown in Fig. 10, indicate that phase noise levels lower than $-60 \mathrm{dBc} @$ $100 \mathrm{~Hz}$ off-set frequency have no significant impact on the precision of the analyzed methods.

Finally, apart from oscillator phase noise effects, it should be emphasized, as mentioned in the previous section, that during the time period required by the traditional STDCC method to generate a single channel estimate the other sounding methods are able to generate several channel estimates snapshots. This advantage is particularly important for the sounding and statistical characterization of time varying channels. 


\section{REFERENCES}

[1] B. Sklar, "Rayleigh fading channels in mobile digital communication systems, Part II: Mitigation", IEEE Communications magazine, pp. 102-109, July, 1997.

[2] C. E. S. Ferreira, "Comparison among wideband mobile radio channel sounding techniques in the presence of sounder imperfections", Doctoral thesis, Electrical Engineering Department, Pontifical Catholic University of Rio de Janeiro, 2013.

[3] J.D. Parsons, D.A. Demery, A.M.D. Turkmani, "Sounding techniques for wideband mobile radio channels: a review", IEE Proceedings-1, Vol. 138, No.5, October 1991.

[4] D. B. Leeson, "A simple model of feedback oscillator noise spectrum", Proceedings of the IEEE, volume 54, issue 2, pg 329-330, 1966.

[5] G. Sauvage, "Phase noise in oscillators: A mathematical analysis of Leeson's model", IEEE Transactions on Instrumentation and Measurements, vol. IM-26, NO. 4, 1977.

[6] X. Huang, F. Tan, W. Wei, and W. Fu, "A revisit to phase noise model of Leeson", Frequency control Symposium, 2007 joint with the 21st European Frequency and Time Forum, IEEE International, pg. 238-241, 2007.

[7] N. J. Kasdin,"Discrete simulation of colored noise and stochastic processes and 1/fa power law noise generator", Proceedings of the IEEE, , Volume: 83, Issue: 5, Page(s): 802 - 827, May 1995.

[8] A. G. Armada, "Understanding the effects of phase noise in orthogonal frequency division multiplexing (OFDM)", IEEE Transactions on Broadcasting, Volume: 47, Issue: 2, Page(s): 153 - 159, Jun 2001.

[9] C. D. Isklander, "A MATLABß-based object-oriented approach to multipath fading channel simulation", Hi-Tek Multisystems, Québec, Canada, http://www.mathworks.com/matlabcentral/filleexchange/18869-a-matlab-based-objectoriented-approach-to-multipath-fading-channel-simulation, 21 Feb 2008.

[10] Ryan J. Pirkl, Gregory D. Durgin, "Optimal sliding correlator channel sounder design”, IEEE Transactions on Wireless Communications, Vol. 7, NO. 9, September 2008

[11] M. J Gans, "A power-spectral theory of propagation in the mobile-radio environmen”, IEEE Transactions on Vehicular Technology;Vol. 21, Iss. 1, pp. 27-38, 1972.

[12] B. Muquet, Z. Wang, G. B. Giannakis, M. de Courville, P. Duhamel, "Cyclic Prefixing or Zero Padding for Wireless Multicarrier Transmissions?", IEEE Transactions on Communications, Vol. 50, NO. 12, Pages 2136-2148, December 2002 


\section{APPENDIX}

\section{Wideband sounding}

Wideband radio channel characterization comprises both the quantity of multipath components present in a particular environment and the way in which they affect the reception, each with their own delay, amplitude and phase. This information creates a time-delay profile.

The sounding of the wideband radio channel is thus an essential tool for systems development and there are different ways to implement it. Alternatives for the construction of wideband sounders are presented, aiming at the increase in the precision of the estimated parameters.

\section{Theoretical Basis of Pulse Compression}

The analysis of pulse compression systems is based on linear systems theory. It is known that if a white noise signal $\mathrm{n}(\mathrm{t})$ is applied to the input of a linear time-invariant system and the output $w(t)$ is correlated with a delayed input replica $n(t-\tau)$, the resulting cross-correlation will be proportional to the impulse response $h(\tau)$ of the system, evaluated in the time delay $\tau$. The white noise autocorrelation function is given by

$$
\mathbb{E}\left[n(t) n^{*}(t-\tau)\right]=R_{n}(\tau)=N_{0} \delta(\tau)
$$

where $\mathbb{E}$ denotes the expected value operator or expectation, (.)* represents the complex conjugate, $R_{n}(\tau)$ is the autocorrelation function of the process $n(t), N_{0}$ is the single-sideband noise power spectral density, and $\delta($.$) is the Dirac delta function. In time domain, a continuous output signal w(t)$ is obtained through the convolution integral

$$
w(t)=\int h(\xi) n(t-\xi) d \xi
$$

Thus, the cross-correlation between the output and delayed input is given by:

$$
\begin{gathered}
\mathbb{E}\left[w(t) n^{*}(t-\tau)\right]=\mathbb{E}\left[\int h(\xi) n(t-\xi) n^{*}(t-\tau) d \xi\right] \\
=\int h(\xi) R_{n}(\tau-\xi) d \xi \\
=N_{0} h(\tau)
\end{gathered}
$$

Therefore, the impulse response of a linear system can be estimated by using a source of white noise and a method to process the correlation between the output signal and the delayed input test signal.

In practice, the systems that apply pulse compression use deterministic waveforms with autocorrelation characteristics similar to the white noise autocorrelation. An easily generated and widely used sequence is the maximum length pseudorandom binary sequence, also known as the pseudo-noise sequence (PN sequence).

\subsection{Matched Filters}

In the sections to follow, lower-case letters in bold represent column vectors, whereas capital letters in bold represent matrices. (.)* denotes the complex conjugate, $(.)^{T}$ indicates transposition, and ( ${ }^{*}$ ) 
represents the quantities in the frequency domain, as opposed to quantities in the time domain, such as the coefficients of the channel impulse response or the samples that are effectively carried through waveforms transmitted in the propagation channel. The symbol $\otimes$ denotes circular convolution and the symbol $*$ denotes linear convolution.

The matched filter sounding method should be considered as a digital method. A known sequence of test that has autocorrelation similar to that of white noise is used. For the remainder of this appendix, the PN sequence is adopted as the test signal to be transmitted. A finite impulse response FIR filter whose impulse response corresponds to the transmitted PN sequence reversed in time, i.e., a filter matched to the test signal, is used in the receiver.

The periodic test sequence represented by $a_{N}(n)$, whose period has length $N$ and duration $N T_{S}$, were $T_{s}$ represents the duration of each symbol can be identified as the output of a Binary Phase Shift Keying BPSK modulator whose input is a pseudorandom binary sequence of length $N$, the elements of which are zeroes and ones obeying their laws of formation. Assuming the amplitude of the BPSK symbols to be equal to \pm 1 , a single period of the periodic test sequence can be represented by the block:

$$
\boldsymbol{a}_{N}=[a(0), \cdots, a(N-1)]^{T}, a(n)= \pm 1, \quad n=0,1, \cdots N-1
$$

For the spectrum shaping of the signal to be transmitted, the classical theory represents a linear modulated channel in baseband. An ideal transmission system is displayed in Fig. A.1, in which necessary changes were introduced to represent the sounding method named "Matched Filter."

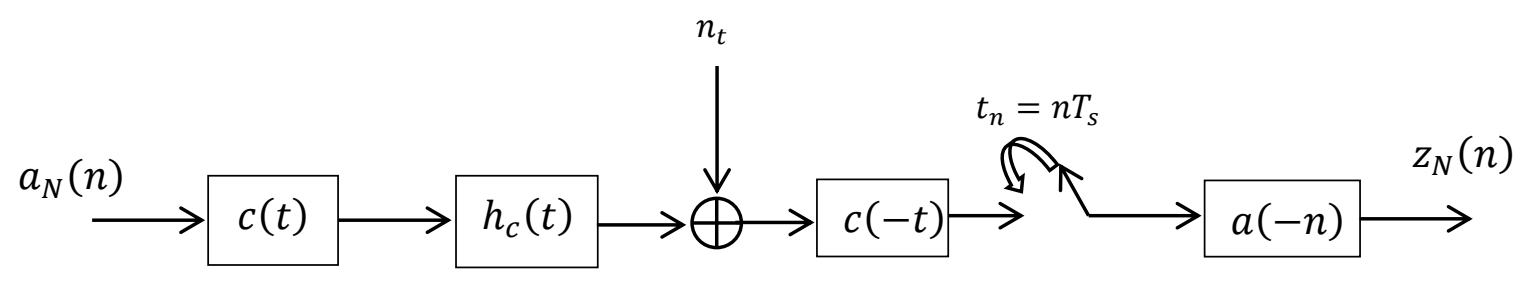

Fig. A.1 - Block diagram of matched filter sounders

In Fig. A.1, the block identified by $c(t)$ represents the transmission square-root-raised-cosine pulse shape filter, the $h_{c}(t)$ block represents the propagation channel impulse response, the $c(-t)$ block represents the receiver filter, which is matched to the transmission filter, and $n_{t}$ is the white noise present at the input of the receiver. Following these blocks, a sampler operating at symbol rate takes samples of the analog output of the receiver filter. Finally, the Matched Filter is inserted, which is matched to the test sequence and is indicated by block $a(-n)$.

Let $h(t)$ be the analog system impulse response, given by the convolution of the shaping transmission filter with the channel impulse response and with the receiver filter, them the discrete time system impulse response is given by:

$$
h(n)=h\left(n T_{s}\right)=\left.c(t) * c(-t) * h_{c}(t)\right|_{t_{n}=n T_{s}},
$$

In the absence of noise in the input of the receiver, the output of the filter matched to the test sequence is represented by: 


$$
z_{N}(n)=a_{N}(n) * a(-n) * h(n)
$$

Considering that the radio channel varies slowly enough to not change significantly during the transmission of a PN sequence, and since the test sequence is periodic, the convolution indicated in (A.6) can be considered to be circular. Denoting by $z_{n}^{(i)}$ the segment of $z_{N}(n)$ resulting from the $i$-th repetition of the test sequence $a$, we have:

$$
z_{n}^{(i)}=a(n) \otimes a(-n) * h^{(i)}(n), \quad n=0,1, \cdots N-1,
$$

In addition, considering that $R_{a}(n)=a(n) \otimes a(-n)$ is the circular autocorrelation of the PN sequence, and knowing that:

$$
R_{a}(n)= \begin{cases}N ; & n=0 \\ -1 ; & n=1,2, \cdots N-1\end{cases}
$$

then $R_{a}(n)$ can be represented as:

$$
R_{a}(n)=(N+1) \delta(n)+f(n), \quad f(n)=-1, n=0,1, \cdots N-1
$$

where $\delta(n)$ is the discrete time impulse defined by:

$$
\delta(n)= \begin{cases}1, & n=0 \\ 0 & n \neq 0\end{cases}
$$

Therefore from (A.7) and (A.8) it results

$$
z_{n}^{(i)}=[(N+1) \delta(n)+f(n)] * h^{(i)}(n)=[N+1] h^{(i)}(n)-\tilde{h}^{(i)}(0)
$$

where $\tilde{h}^{(i)}(0)$ is the value of the discrete system frequency response at zero frequency resulting from

$$
f(n) * h^{(i)}(n)=\sum_{k=0}^{L-1} h^{(i)}(k) f(n-k)=-\sum_{k=0}^{L-1} h^{(i)}(k)=-\tilde{h}^{(i)}(0)
$$

where $f(n-k)=-1$ and $L$ represents the length of the response of the system to a discrete impulse.

From (A.9), we have that:

$$
\frac{z_{n}^{(i)}}{N+1}=h^{(i)}(n)-\frac{\widetilde{h}(0)}{N+1}
$$

and assuming that the PN sequence is sufficiently long:

$$
h^{(i)}(n) \cong \frac{z_{n}^{(i)}}{N+1} \cong \frac{z_{n}^{(i)}}{N}
$$

Finally, we observe that:

- $\quad N$ must be greater than the span of $h^{(i)}(n)$ to avoid superposition $(N>L)$

- Regarding the resolution the time interval between the paths of $h_{c}(t)$ must be greater than the span of $c(t) * c(-t)$.

\section{OFDM}

\subsection{Notations}

In this section, subscripts are sometimes used to emphasize dimensions of vectors and matrices. For example, $\mathbf{s}_{\mathrm{M}}$ indicates an $\mathrm{M} \times 1$ vector, while $\mathbf{W}_{\mathrm{M}}$ indicates an $\mathrm{M} \times \mathbf{M}$ matrix. The vector elements and matrices are indexed by one or two of those subscripts, respectively, such as, for example, $s_{k}$ or $W_{k, l}$. 
Matrix $\mathbf{W}_{\mathrm{M}}$ implements the Discrete Fourier Transform (DFT) of size $\mathbf{M}$, whose elements are $\mathrm{W}_{\mathrm{k}, \mathrm{l}}=\frac{1}{\sqrt{\mathrm{M}}} \mathrm{e}^{-\mathrm{j} 2 \pi \mathrm{kl} / \mathrm{M}}$. The $\odot$ symbol represents the product of two vectors, term by term, and $(.)^{\mathrm{H}}$ indicates the Hermitian transposition.

\section{$2.2 \quad$ OFDM sounder}

The idea behind the OFDM transmission technique using the so called cyclic prefix is to make the linear convolution of the transmitted signal with the channel impulse response equivalent to a circular convolution. The resulting circular convolution is then transformed into a multiplication in the frequency domain. The principle behind this operation is shown in Fig. A.2. In this figure, the difference between circular and linear convolution is illustrated for the case of the repeated transmission of blocks of symbols, $\mathbf{s}(i)$. The circular convolution of the periodic sequence of blocks $\mathbf{s}(i)$ with the channel impulse response $\mathbf{h}$ that results in $\mathbf{r}(i)$ is displayed in Fig. A.2(a). In the frequency domain, we than have:

$$
\operatorname{DFT}(\mathbf{r}(i))=\operatorname{DFT}(\mathbf{h}) \times \operatorname{DFT}(\mathbf{s}(i))
$$

It becomes clear from the superimposition in the beginning of the block that the result of the linear convolution of $[\ldots, \mathbf{s}(i-1), \mathbf{s}(i), \mathbf{s}(i+1), \ldots]$ with $\mathbf{h}$ is, in general, not equal to the circular convolution. In fact, there is no reason for the end of the $\mathbf{s}(i-1)$ block to be equal to the end of the $\mathbf{s}(i)$ block. In addition, the output of the channel corresponding to the block $\mathbf{s}(i)$ is superimposed onto the output of the previous block $(i-1)$, the so called interblock interference. The cyclic prefix principle introduces redundancy in the transmitted signal in such a way that the superimposition induced by the channel memory corresponds to that of the circular convolution. In this way, the block of symbols $\mathbf{r}(i)$ that correspond to the transmission of $\mathbf{s}(i)$ is exactly the same as the circular convolution of $\mathbf{s}(i)$ and $\mathbf{h}$. Therefore, $\mathbf{s}(i)$ is easily recovered from the received $\mathbf{r}(i)$ block by performing a DFT. Furthermore, $\mathbf{r}(i)$ becomes independent from the $\mathbf{s}(i-1)$ block, which is important since the recovery of $\mathbf{s}(i)$ does not require the knowledge of previously sent symbols. When OFDM is used as a sounding method the insertion of a cyclic prefix is not necessary since the transmitted blocks are all equal, that is $\mathbf{s}(i)=\mathbf{s}, \forall i$. Therefore the linear convolution of $\mathbf{h}$ with the periodic sequence formed by the $\mathbf{s}$ block repetition generates a sequence formed by the repetition of $\mathbf{r}=\mathbf{h} \otimes \mathbf{s}$, as shown in Fig. A.2a. 
a-Circular

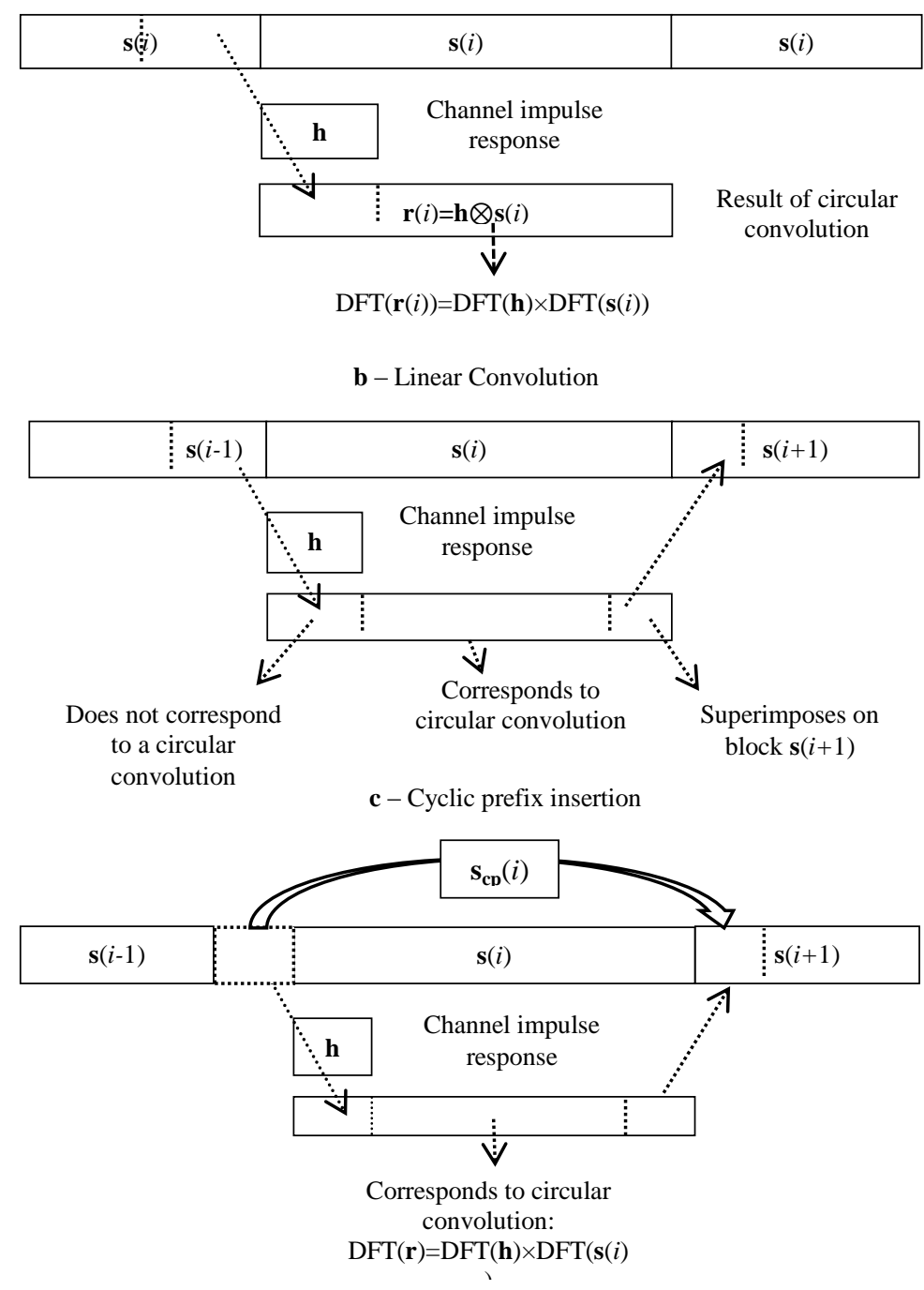

Fig. A.2 - Cyclic prefix principle

The implemented OFDM system used as a sounding method of the radio mobile channel is based on the OFDM principle, whose transceiver scheme is described in Fig. A.3. This figure represents the baseband equivalent in the discrete time domain. In theory, it is assumed that both the transmitted and received signals are sampled in synchrony with the system basic rate, and the focus will be on OFDM discrete time aspects.

In the baseband equivalent model of the OFDM sounder transceiver, the complex symbols representing the points of the signal constellation of the adopted modulation are transmitted in blocks of length $\mathrm{M}: \tilde{\mathbf{s}}_{\mathrm{M}}=\left[\tilde{s}_{0}, \ldots, \tilde{s}_{M-1}\right]^{T}$ that forms the test sequence. These symbols are initially transformed by the IDFT unity matrix $\mathbf{W}_{\mathrm{M}}^{\mathrm{H}}$, whose elements are $\exp \{+j 2 \pi m k / M\} / \sqrt{M}$, which transforms the $\tilde{\mathbf{s}}_{M}$ block to the time domain now denoted by $\mathbf{s}_{M}$ block. The channel is represented by its equivalent discrete time domain model, and its effect is modeled as a time variant linear filter with a size $L$ finite impulse response FIR, represented by the impulse response $\mathbf{h}(i)=\left[h_{0}(i), \ldots, h_{L-1}(i)\right]^{T}$ 
where $L-1$ is the channel order. It is assumed here that the channel does not change that during the test sequence transmission time. This is a fair approximation if the product $f_{D} \times T \leq 10^{-4}$ where $f_{D}$ is the maximum Doppler shift and $T$ is the test sequence duration. Since the test sequence length is made bigger than the channel order, $(M>L-1)$, the mobile radio channel can be estimated without ambiguity. The length $M$ impulse response $\mathbf{h}_{\mathrm{M}}(i)$ is defined as $\mathbf{h}(i)$ appended with zeroes until the length $M$ is reached.

$$
\begin{array}{llll}
\tilde{\mathbf{s}}_{M} & \mathbf{s}_{M} & \mathbf{r}_{M}(i) & \tilde{\mathbf{r}}_{M}(i)
\end{array}
$$

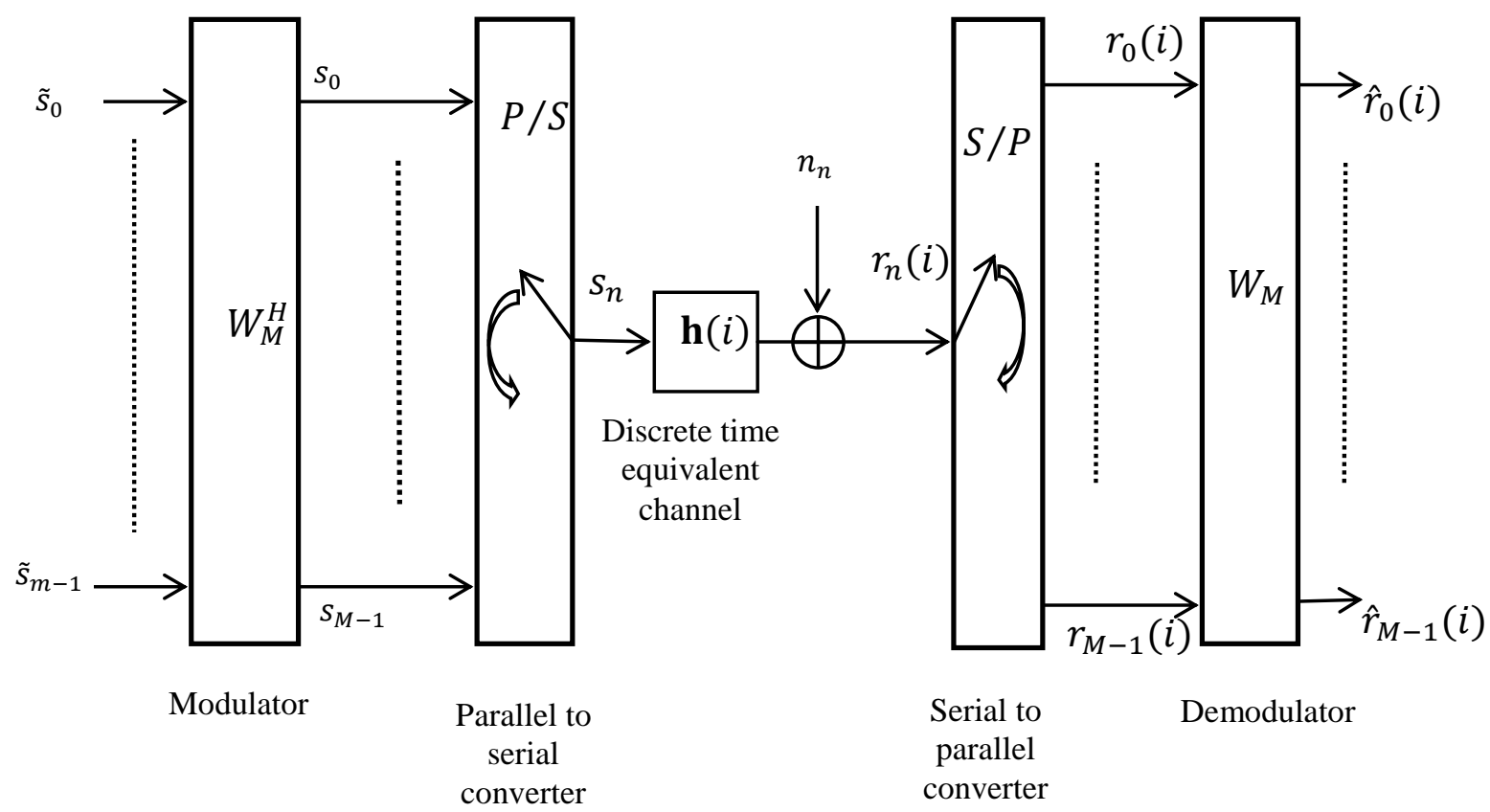

Fig. A.3 - Discrete model for an OFDM conventional transceiver

The size $M$ test sequence $\widetilde{\mathbf{s}}_{\mathrm{M}}$ is IDFT transformed by the matrix $\mathbf{W}_{\mathrm{M}}^{\mathrm{H}}$. In time domain the transmitted block of symbols is then $\mathbf{s}_{\mathrm{M}}=\mathbf{W}_{\mathrm{M}}^{\mathrm{H}} \widetilde{\mathbf{s}}_{\mathrm{M}}$. The received block of symbols $\mathbf{r}_{\mathrm{M}}(i)$ corresponds to the circular convolution between $\mathbf{s}_{\mathrm{M}}$ and $\mathbf{h}(i)$ plus the noise contribution $\mathbf{n}_{\mathrm{M}}$ where $\mathbf{n}_{\mathrm{M}}=$ $\left[\left(n_{0}, \ldots n_{M-1}\right)\right]^{T}$ represents a vector of complex noise samples in the receiver's baseband equivalent model of the sounder. Hence, the received time domain vector can be expressed by:

$$
\mathbf{r}_{\mathrm{M}}(i)=\mathbf{h}_{\mathrm{M}}(i) \otimes \mathbf{s}_{\mathrm{M}}+\mathbf{n}_{\mathrm{M}}
$$

The circular convolution presented in equation (A.14) may be expressed in matrix form:

$$
\left[\begin{array}{c}
r_{0}(i) \\
\vdots \\
r_{L}(i) \\
\vdots \\
\vdots \\
r_{M-1}(i)
\end{array}\right]=\left[\begin{array}{cccccc}
h_{0}(i) & 0 & \cdots & h_{L}(i) & \cdots & h_{1}(i) \\
\vdots & \ddots & \ddots & \vdots & \ddots & \vdots \\
h_{L-1}(i) & \vdots & \ddots & \ddots & \ddots & h_{L}(i) \\
0 & \ddots & \vdots & \ddots & \ddots & \vdots \\
\vdots & \ddots & \ddots & \vdots & \ddots & 0 \\
0 & \cdots & 0 & h_{L-1}(i) & \cdots & h_{0}(i)
\end{array}\right]\left[\begin{array}{c}
s_{0} \\
\vdots \\
s_{L} \\
\vdots \\
\vdots \\
s_{M-1}
\end{array}\right]+\left[\begin{array}{c}
n_{0} \\
\vdots \\
n_{L} \\
\vdots \\
\vdots \\
n_{M-1}
\end{array}\right]
$$

The elements of each matrix column are obtained shifting the elements of the previous column circularly by one position. Since this matrix is a circulant matrix $\mathbf{C}_{\mathrm{M}}\left(\mathbf{h}_{\mathrm{M}}\right)$ it can be diagonalized by 
pre- and post-multiplication by unitary DFT and IDFT matrices. Therefore,

$$
\begin{array}{r}
\tilde{\mathbf{r}}_{M}(i)=\mathbf{W}_{M} \mathbf{r}_{M}(i)=\mathbf{W}_{M} \mathbf{C}_{M}\left(\mathbf{h}_{M}\right) \mathbf{s}_{M}+\mathbf{W}_{M} \mathbf{n}_{M}= \\
\mathbf{W}_{M} \mathbf{C}_{M}\left(\mathbf{h}_{M}\right) \mathbf{W}_{M}^{H} \widetilde{\mathbf{s}}_{M}+\mathbf{W}_{M} \mathbf{n}_{M}=\operatorname{diag}\left(\tilde{\mathbf{h}}_{M}(i)\right) \tilde{\mathbf{s}}_{M}+\widetilde{\mathbf{n}}_{M}
\end{array}
$$

In (A.16), $\operatorname{diag}\left(\tilde{\mathbf{h}}_{\mathrm{M}}(i)\right)$ represents a $\mathrm{M} \times \mathrm{M}$ diagonal matrix whose elements are the components of the $\mathbf{h}_{\mathrm{M}}(i)$ vector transformed to the frequency domain $\tilde{\mathbf{h}}_{\mathrm{M}}(i)=\sqrt{\mathrm{M}} \mathbf{W}_{\mathrm{M}} \mathbf{h}_{\mathrm{M}}(i)$, whereas $\widetilde{\mathbf{n}}_{\mathrm{M}}=$ $\mathbf{W}_{M} \mathbf{n}_{M}$ is the noise vector at the receiver imput transformed to the frequency domain. The equation (A.16) can be rewritten as:

$$
\tilde{\mathbf{r}}_{M}(i)=\widetilde{\mathbf{h}}_{M}(i) \odot \widetilde{\mathbf{s}}_{M}+\widetilde{\mathbf{n}}_{M}
$$

It then results that the $n$-th component of $\tilde{\mathbf{r}}_{\mathrm{M}}(i)$, denoted by $\widetilde{\mathbf{r}}_{\mathrm{M}_{\mathrm{n}}}(i)$, can be independently detected from the others without intersymbol interference between adjacent test sequence symbols. Expression (A.17) can be rewritten as:

$$
\tilde{\mathbf{r}}_{M_{n}}(i)=\tilde{\mathbf{h}}_{M_{n}}(i) \tilde{\mathbf{s}}_{M_{n}}+\widetilde{\mathbf{n}}_{M_{n}}
$$

where $\tilde{\mathbf{h}}_{\mathrm{M}_{\mathrm{n}}}(i)$ and $\widetilde{\mathbf{n}}_{\mathrm{M}_{\mathrm{n}}}(i)$ are the nth components of the channel frequency response $\tilde{\mathbf{h}}_{\mathrm{M}}(i)$ and the noise vector $\widetilde{\mathbf{n}}_{\mathrm{M}}(i)$, respectively. Since the test sequence is known, the estimation of the channel frequency response at the receiver is obtained by dividing each element of the received signal by each element of the test signal. Observing that $\operatorname{diag}^{-1}\left(\widetilde{\mathbf{s}}_{\mathrm{M}}\right)$ is an $\mathrm{M} \times \mathrm{M}$ diagonal matrix that contains the reciprocal of each $\tilde{\mathbf{s}}_{\mathrm{M}}$ element and the channel is considered time invariant during each test sequence transmission, an estimate of the channel impulse response is given by:

$$
\hat{\mathbf{h}}_{M}(i)=\operatorname{diag}^{-1}\left(\widetilde{\mathbf{s}}_{M}\right) \tilde{\mathbf{r}}_{M}(i)=\tilde{\mathbf{h}}_{M}+\operatorname{diag}^{-1}\left(\widetilde{\mathbf{s}}_{M}\right) \widetilde{\mathbf{n}}_{M}(i)
$$

It is clear that the noise present at the receiver will affect the accuracy of the channel estimation.

Finally, the estimated impulse response of the sounded channel is given by:

$$
\mathbf{h}_{M}(i)=\frac{1}{\sqrt{M}} \mathbf{W}_{M}^{H} \hat{\tilde{\mathbf{h}}}_{M}(i)
$$

